\title{
AN ISOPERIMETRIC INEQUALITY
}

\author{
F. J. ALMGREN, JR.
}

If $f$ maps the $k$-cube $I^{k}$ into $R^{n}$ so that the images of opposite $k-1$ faces of $I^{k}$ are at least distance $b$ apart, it is plausible that the volume of $f$ can be no smaller than $b^{k}$. This is true if $f$ is Lipschitzian and is established as a corollary to the more general theorem below. This problem was suggested by D. C. Spencer, who together with S. Bergman proved it for $k=2$ in [2].

DEFINITIONS.

(1) $I_{*}\left(R^{n}\right)=\oplus_{j} I_{j}\left(R^{n}\right)$ is the chain complex of integral currents in $R^{n}$ with boundary homomorphism $\partial$ as defined in [3]. The mass of a current $T$ is written $M(T)$. For $T \in I_{0}\left(R^{n}\right)$ let $M_{0}(T)$ be the absolute value of the coefficient sum of $T$.

(2) $g^{k}$ is the cell complex of the $k$-cube $I^{k}[1 ; 2.1]$, i.e., the chain complex generated by the cubical faces of $I^{k}$ of various dimensions. $g^{1}$ is generated by $\{[0,1],[0],[1]\}$ with $d[0,1]=[1]-[0], d[0]$ $=d[1]=0 . g^{2}$ is generated by $\{[0,1] \otimes[0,1],[0,1] \otimes[1],[0] \otimes[0]$, $\cdots\}$, etc. Let $\alpha^{k}=[0,1] \otimes \cdots \otimes[0,1]$ be the unique $k$ cell in $\mathfrak{g}^{k}$, and let $\alpha^{k}(i, \epsilon), i=1,2, \cdots, k, \epsilon=0,1$ denote the $k-1$ cell obtained by setting the $i$ th coordinate equal to $\epsilon$.

(3) For $A \subset R^{n}$ define $u_{A}: R^{n} \rightarrow R, u_{A}(x)=$ distance $(x, A)$. Set $U_{r}=R^{n} \cap\left\{x: u_{A}(x)<r\right\}$ for $r \in R$. Note that $u_{A}$ satisfies a Lipschitz condition with constant 1 for any $A$.

Theorem. Let $F: g^{k} \rightarrow I_{*}\left(R^{n}\right)$ be a chain map of degree 0 such that

(1) For some 0 cell (vertex) $v \in \mathfrak{g}^{k}, M_{0}(F(v)) \geqq 1$.

(2) $\inf \{|x-y|: x \in$ support $(F(\beta)), y \in$ support $(F(\gamma))\} \geqq b_{i}$ whenever $\beta$ is a face of $\alpha^{k}(i, 0)$ and $\gamma$ is a face of $\alpha^{k}(i, 1)$ for some $i=1,2$, $\cdots, k$.

Then $M\left(F\left(\alpha^{k}\right)\right) \geqq M_{0}(F(v)) \cdot \prod_{i=1}^{k} b_{i}$.

Lemma. Let $A$ and $B$ be subsets of $R^{n}$ with $\inf \{|x-y|: x \in A, y \in B\}$ $=b$. Suppose $S, T \in I_{0}\left(R^{n}\right)$, support $(S) \subset A$, support $(T) \subset B$, and $M_{0}(S) \geqq 1$. Suppose also $Q \in I_{1}\left(R^{n}\right)$ with $\partial Q=S-T$. Then $M_{0}(S)$ $=M_{0}(T)$ and $M(Q) \geqq M_{0}(S) \cdot b$. Also, for $L_{1}$ almost all $r \in(0, b)$, $\partial\left(Q \cap U_{r}(A)\right)-\partial Q \cap U_{r}(A) \in I_{0}\left(R^{n}\right)$ and

$$
M_{0}\left(\partial\left(Q \cap U_{r}(A)\right)-\partial Q \cap U_{r}(A)\right)=M_{0}(S) .
$$

Received by the editors January 7, 1963. 
Proof. The lemma follows in part from [3, 3.8(3), 3.9, 3.10, 8.14] and the rest is obvious.

Proof of The TheOREM. By induction. For $k=1$, the theorem is implied by the lemma. Assume the theorem holds up to dimension $k-1$. Consider the map

$$
\begin{aligned}
G_{r}: I^{k-1} & \rightarrow I_{*}\left(R^{n}\right) \\
G_{r}(\beta) & =\partial\left[F([0,1] \otimes \beta) \cap U_{r}\left(\operatorname{support}\left[F\left(\alpha^{k}(1,0)\right)\right]\right)\right] \\
& -\partial F([0,1] \otimes \beta) \cap U_{r}\left(\operatorname{support}\left[F\left(\alpha^{k}(1,0)\right)\right]\right)
\end{aligned}
$$

for each cell $\beta \in g^{k-1}$. By $[3 ; 3.8(3), 3.9,3.10,8.14], G_{r}$ is defined for $L_{1}$ almost all $r \in\left(0, b_{1}\right)$. One verifies that for each such $r, G_{r}$ is a chain map. Using the lemma, it follows that each such $G_{r}$ satisfies the induction hypothesis. Thus

$$
M\left(G_{r}\left(\alpha^{k-1}\right)\right) \geqq M_{0}(F(v)) \cdot \prod_{i=2}^{k} b_{i}
$$

Using $[3,3.10]$ one sees

$$
M\left(F\left(\alpha^{k}\right)\right) \geqq \int_{0}^{b_{1}} M\left(G_{r}\left(\alpha^{k-1}\right)\right) d r \geqq M_{0}(F(v)) \cdot \prod_{i=1}^{k} b_{i}
$$

CoRollary. Let $f: I^{k} \rightarrow R^{n}$ be Lipschitzian with $|f(x)-f(y)| \geqq b$ whenever $x$ and $y$ lie on opposite $k-1$ dimensional faces of $I^{k}$. The restrictions of $f$ to the various faces of $I^{k}$ determine integral currents in $R^{n}$, and since volume is always greater than or equal to mass, one has

$$
\operatorname{volume}(f) \geqq M\left(f_{\sharp}\left(I^{k}\right)\right) \geqq b^{k} \text {. }
$$

\section{REFERENCES}

1. F. J. Almgren, Jr., The homotopy groups of the integral cycle groups, Topology 1 (1962), 257-299.

2. S. Bergman and D. C. Spencer, A property of pseudo-conformal transformations in the neighborhood of boundary points, Duke Math. J. 9 (1942), 757-762.

3. H. Federer and W. H. Fleming, Normal and integral currents, Ann. of Math. (2) 72 (1960), 458-520.

Princeton UNIVERSity 